\title{
Combined use of thyroid stimulating hormone plus free thyroxine levels and gestational age at birth for the prediction of neonatal hypothyroidism and associated risk factors
}

\author{
JUNQI LI ${ }^{1}$, JING CHENG ${ }^{1}$ and QIUYUE LI $^{2}$ \\ ${ }^{1}$ Department of Obstetrics, The First Affiliated Hospital of Chongqing Medical University, Chongqing 400016; \\ ${ }^{2}$ Department of Obstetrics, The Second Affiliated Hospital of Chongqing Medical University, Chongqing 400010, P.R. China
}

Received September 10, 2019; Accepted June 10, 2020

DOI: $10.3892 /$ etm.2020.9349

\begin{abstract}
The present study aimed to explore the effectiveness of the combined use of thyroid-stimulating hormone (TSH) and free thyroxine [(FT4); TSH+FT4] levels and gestational age at birth to predict neonatal hypothyroidism and to identify the risk factors associated with the disease. The clinical data of 686 neonates with suspected hypothyroidism (TSH >10 mIU/l) who were admitted to The First Affiliated Hospital of Chongqing Medical University were retrospectively analyzed. From these, 70 neonates with confirmed hypothyroidism were assigned to the patient group and another 70 neonates with normal thyroid function to the normal (control) group. Sex, gestational age at birth, Apgar score, birth weight, body length, head circumference and heart rate data were collected. TSH and FT4 levels were measured by electrochemiluminescence immunoassay, and the predictive value of combined use of TSH+FT4 and gestational age at birth on neonatal hypothyroidism was analyzed. The prespecified secondary outcomes were the risk factors for neonatal hypothyroidism determined using univariate and logistic regression analyses. TSH levels were significantly higher, whereas FT4 levels were lower in the patient group at 3 days of age compared with the control group $(\mathrm{P}<0.05)$. After 8 -week treatment with thyroxine, these levels were not significantly different between the patient and control groups $(\mathrm{P}<0.05)$. The combined use of TSH+FT4 levels and gestational age at birth to predict neonatal hypothyroidism demonstrated a significantly improved sensitivity, specificity, accuracy, positive predictive value and negative predictive value $(92.86,97.26,96.76,81.25$ and $99.07 \%$, respectively) compared with the use of only TSH+FT4 levels $(\mathrm{P}<0.05)$. Logistic regression analysis revealed a low gestational age
\end{abstract}

Correspondence to: Dr Junqi Li, Department of Obstetrics, The First Affiliated Hospital of Chongqing Medical University, 1 Youyi Road, Chongqing 400016, P.R. China

E-mail: jun8686y@163.com

Key words: thyroid-stimulating hormone, free thyroxine, gestational age at birth, neonate, hypothyroidism, risk factors at birth, maternal thyroid dysfunction and low birth weight were risk factors for neonatal hypothyroidism $(\mathrm{P}<0.05)$. The combined use of TSH+FT4 levels and gestational age at birth resulted in an improved prediction of neonatal hypothyroidism and contributed to early therapeutic intervention. Thus, special intervention is necessary for pregnant women with thyroid dysfunction to reduce the incidence of neonatal hypothyroidism.

\section{Introduction}

Thyroid hormones play a critical role in the differentiation and proliferation of neurons in the brain and are closely related to brain development (1). Hypothyroidism is a thyroid dysfunction caused by insufficient synthesis, secretion or physiological effects of thyroid hormones, which is characterized by an increase in serum levels of thyroid-stimulating hormone (TSH) accompanied by a reduction in the level of free thyroxine (FT4) and in some cases a reduction in the level of free triiodothyronine (FT3) (2). Neonatal hypothyroidism may cause growth and developmental disorders as well as mental retardation in neonates $(3,4)$. Neonatal hypothyroidism is a common clinical condition and is one of the major causes of mental and physical retardation in children (5). Neonatal screening in China in 2,000 indicated that the incidence of neonatal hypothyroidism was approximately $1 / 3,624$ in and that the condition severely endangers the health of neonates and can cause irreversible damage following the onset of clinical symptoms $(6,7)$. The pathogenesis and causes of neonatal hypothyroidism remain unclear; however, it is reported that pregnant women's health and lifestyle and the foetal environment are factors influencing development of hypothyroidism (8). Thyroid function tests are important methods for early detection and intervention in neonatal hypothyroidism. The early detection of neonatal hypothyroidism facilitates therapeutic intervention before the onset of clinical symptoms and enables optimal physical and intellectual development in neonates (9). The risk factors for neonatal hypothyroidism vary and have been reported to include increased gestational age at birth and birth weight. Neonates who had a premature birth, low birth weight, post-term birth and fetal macrosomia are at a higher risk of hypothyroidism compared with neonates born at term (10-12). 
In previous studies, neonatal hypothyroidism screening was mainly performed by measuring thyroid-stimulating hormone (TSH) and free thyroxine [(FT4); TSH+FT4] levels, which had low sensitivity and specificity (13). Zhang et al (14) showed that most neonatal hyperthyrotropinemia is resolved with age, but some infants will continue to have abnormal thyroid function, and should be actively followed up to prevent hypothyroidism (14). Newborn disease screening is widely performed and term neonates with congenital hypothyroidism can be diagnosed in a timely manner. However, there are still no accurate diagnostic criteria for abnormal thyroid function in premature infants. Premature infants have clinical manifestations of hypothyroidism, such as lethargy, anorexia, fatigue, constipation, delayed jaundice, edema, dry skin and poor peripheral circulation. Without timely intervention, hypothyroidism may have adverse effects on neonates' growth, development, cognition and even cause irreversible brain damage (15).

Based on this, in the present study, the risk factors for neonatal hypothyroidism using gestational age at birth in combination with TSH+FT4 levels were investigated to screen for neonatal hypothyroidism and predict its occurrence.

\section{Materials and methods}

Study subjects. In total, 686 neonates with suspected hypothyroidism (TSH $>10 \mathrm{mIU} / \mathrm{l}$ ) who were admitted to the The First Affiliated Hospital of Chongqing Medical University (Chongqing, China) between January 2012 and January 2019 were included in this study for retrospective analysis. From these, 70 with confirmed hypothyroidism were randomly assigned to the patient group, including 30 males and 40 females with an average age of $37.26 \pm 4.35$ weeks. Hypothyroidism diagnosis was performed using the following criteria provided by the Clinical Laboratory of The First Affiliated Hospital of Chongqing Medical University: i) increased serum TSH level (normal TSH level, 0.35-5.50 $\mu \mathrm{IU} / \mathrm{ml}$ ); ii) decreased serum FT4 level (normal FT4 level, 0.89-1.76 ng/dl); and iii) normal/decreased free triiodothyronine (FT3) level (normal FT3 level, 2.30-4.20 pg/ml). Neonates who did not meet the above diagnostic criteria for hypothyroidism were excluded from the patient group. In the patient group, the gestational age at birth of 15 neonates was $<37$ weeks, 47 neonates was $37-42$ weeks and 8 neonates was $>42$ weeks. The normal (control) group was comprised of 70 neonates with normal thyroid function, including 36 males and 34 females with an average age of $39.58 \pm 4.23$ weeks. These 70 neonates with normal thyroid function were recruited during the same period as the patient group and were not screened from the 686 aforementioned neonates. The inclusion criteria for the control group neonates were: i) born at The First Affiliated Hospital of Chongqing Medical University; ii) live births; iii) had no congenital malformation; iv) underwent their first examination at the age of $<3$ days; v) parents provided written consent for study participation; and vi) whose mothers underwent thyroid function tests during pregnancy and had a normal range. The exclusion criteria for the control group neonates were: i) Levothyroxine ${ }^{\circledR}$ therapy prior to examination; ii) had metabolic disorders; or iii) had incomplete examination data.
The present study was approved by the Ethics Committee of The First Affiliated Hospital of Chongqing Medical University (Chongqing, China) and signed informed consent was obtained from the parents of all the neonates who were included in the study.

Electrochemiluminescence (ECL) assays and clinical information. Information on indicators (including sex, gestational age at birth, Apgar score, birth weight, body length, head circumference and heart rate) were collected from the neonates in the patient and control groups. TSH and FT4 levels were first measured in neonates $72 \mathrm{~h}$ after birth by the electrochemiluminescence immunoassay using the DIX800 Chemiluminescence Analyzer (Beckman Coulter, Inc.) and FT3 and FT4 chemiluminescence immunoassay kits (access kit; cat. nos. 170611 and 171230) from Beckman Coulter, Inc. In this procedure, drops of blood were collected from the neonatal heel and were tested by filter paper. Venous blood samples $(3 \mathrm{ml})$ were collected from the elbow in tubes containing anticoagulant and centrifuged at 2,264 $\mathrm{x} \mathrm{g}$ for $30 \mathrm{~min}$ at $4^{\circ} \mathrm{C}$ to extract serum.

Treatment regimen. Neonates with confirmed hypothyroidism were orally administered $10-15 \mu \mathrm{g} / \mathrm{kg} / \mathrm{day}$ thyroxine (Shandong Lubei Pharmaceutical Co., Ltd.; SFDA approval no. H3702162). TSH and FT4 levels were re-examined at 2, 4 and 8 weeks of age and compared between the control and patient groups. In addition, data were gathered on maternal thyroid dysfunction, fetal macrosomia and low-birth-weight neonates in the aforementioned groups. Diagnostic criteria for maternal thyroid dysfunction were increased TSH; decreased FT4; and normal or decreased FT3 (16).

Statistical analyses. Data were analyzed using SPSS Statistics v.23.0 (IBM Corp.). Measurement data are expressed as mean \pm standard deviation values using independent samples t-test. Numerical data are presented as percentages and were analyzed using the $\chi^{2}$ test. One-way ANOVA was used to compare the mean among multiple groups and the least significant difference (LSD) post hoc test was used for pairwise comparison when the variance was homogeneous and the Dunnett's T3 post hoc was used when the variance was heterogeneous. The related risk factors for neonatal hypothyroidism were subjected to univariate analysis and logistic regression analysis was performed when univariate analysis demonstrated significant differences. $\mathrm{P}<0.05$ was considered to indicate a statistically significant difference.

\section{Results}

Comparison of clinical and demographic data between the patient and control groups. Sex, gestational age at birth, Apgar score, birth weight, body length, head circumference and heart rate of the two groups were collected and compared and the results showed that there were no significant differences between the patient and control groups in terms of sex, body length, head circumference and delivery mode $(\mathrm{P}>0.05$; Table I). However, the patient group had a significantly lower gestational age at birth, Apgar score, birth weight and heart rate compared with the control group $(\mathrm{P}<0.05$; Table I) 
Table I. Comparison of the clinical and demographic data of neonates with hypothyroidism (patients) and normal (control) neonates.

\begin{tabular}{lccrr}
\hline Parameters & Patient group $(\mathrm{n}=70)$ & Control group $(\mathrm{n}=70)$ & T-test $/ \chi^{2}$ test & P-value \\
\hline Sex (male/female), $\mathrm{n}$ & $30 / 40$ & $36 / 34$ & 1.032 & 0.310 \\
Gestational age (weeks), mean $\pm \mathrm{SD}$ & $37.26 \pm 4.35$ & $39.58 \pm 4.23$ & 3.199 & 0.002 \\
Apgar score (scores), mean \pm SD & $9.06 \pm 0.52$ & $9.53 \pm 0.47$ & 5.610 & $<0.001$ \\
Birth weight (g), mean \pm SD & $3.50 \pm 1.14$ & $3.92 \pm 1.17$ & 2.151 & 0.033 \\
Body length (cm), mean $\pm \mathrm{SD}$ & $48.49 \pm 5.23$ & $50.19 \pm 5.44$ & 1.885 & 0.062 \\
Head circumference $(\mathrm{cm})$, mean $\pm \mathrm{SD}$ & $33.56 \pm 2.36$ & $34.22 \pm 2.28$ & 1.683 & 0.095 \\
Heart rate (bpm), mean \pm SD & $97.22 \pm 10.65$ & $129.71 \pm 10.79$ & 17.930 & $<0.001$ \\
Delivery modes (eutocia/cesarean section), $\mathrm{n}$ & $48 / 22$ & $52 / 18$ & 0.560 & 0.454 \\
\hline
\end{tabular}

Sex and delivery modes are nominal data, which were analyzed by $\chi^{2}$ test, with the statistical value of $\chi^{2}$; other items are measurement data, which were analyzed by T-test, with the statistical value of $t$.
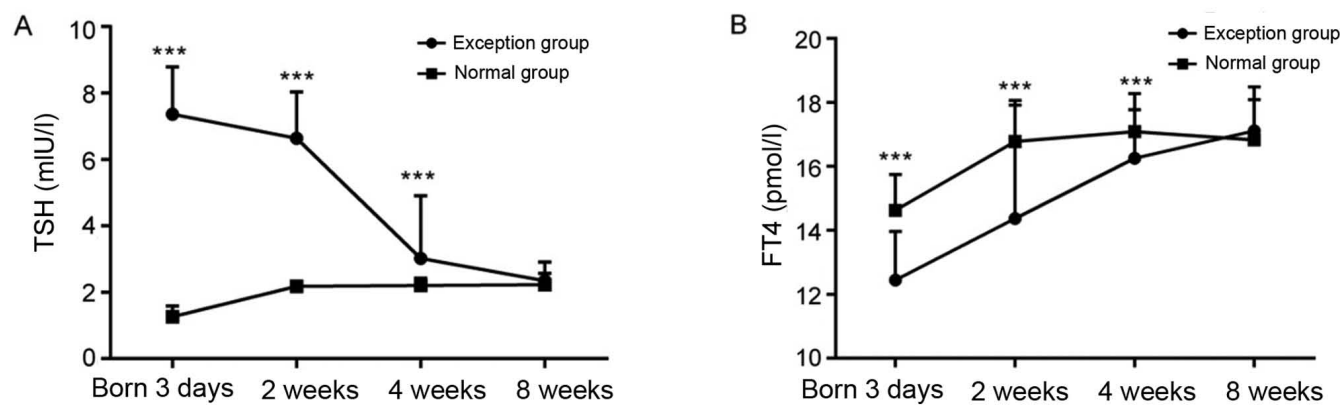

Figure 1. Comparison of TSH and FT4 levels between neonates with hypothyroidism (patients) and normal (control) neonates. (A) The TSH levels in newborns with hypothyroidism are significantly higher at 3 days of age and normalized after 8 weeks of treatment. (B) Newborns with hypothyroidism had significantly lower FT4 levels at 3 days of age that normalized after 8 weeks of treatment. ${ }^{* * *} \mathrm{P}<0.001$ compared with the normal group. TSH, thyroid-stimulating hormone; FT4, free thyroxine.
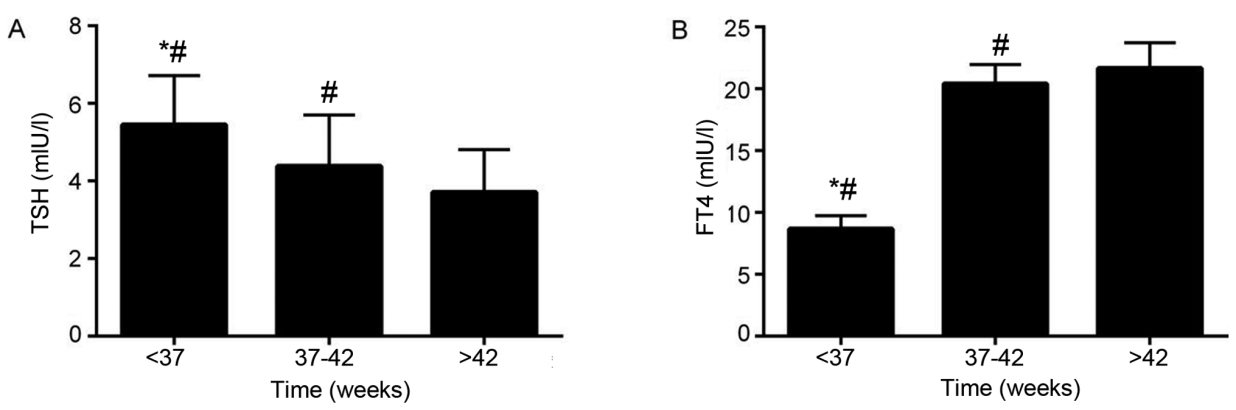

Figure 2. Comparison of TSH and FT4 levels at different gestational ages at birth in neonates with hypothyroidism. In neonates with hypothyroidism, with the increase of gestational age at birth, TSH (A) and FT4 (B) levels were significantly decreased and increased, respectively. "P $<0.05$ compared with $37-42$ weeks; ${ }^{\#} \mathrm{P}<0.05$ compared with $>42$ weeks. TSH, thyroid-stimulating hormone; FT4, free thyroxine.

indicating that the neonates with hypothyroidism had poorer clinical indicators compared with the control neonates.

Neonates with hypothyroidism had higher TSH levels and lower FT4 levels compared with controls. TSH and FT4 levels of the two groups were measured by electrochemiluminescence immunoassay, and the results showed that significantly higher TSH levels but lower FT4 levels at 3 days of age were observed in the patient group compared with the control group $(\mathrm{P}<0.05$; Fig. 1A and $\mathrm{B})$. In addition, the TSH level decreased, however FT4 levels increased during the 8 weeks of treatment (Fig. 1). Following 8 weeks of treatment, there was no significant difference in the TSH and FT4 levels between the patient and control groups ( $\mathrm{P}>0.05$; Fig. 1). Thus, neonates may gradually recover from hypothyroidism diagnosed at birth following treatment.

Effect of different gestational age at birth on TSH and FT4 levels in neonates with hypothyroidism. Neonates with hypothyroidism were divided into $<37$ weeks, 37-42 weeks, and 
Table II. Predictive values for the combined use of gestational age with TSH and FT4 values for screening neonatal thyroid dysfunction.

\begin{tabular}{lcccccc}
\hline $\begin{array}{l}\text { Screening } \\
\text { method }\end{array}$ & $\begin{array}{c}\text { Positive, } \% \\
(\mathrm{n} / \mathrm{n})\end{array}$ & $\begin{array}{c}\text { Sensibility, } \% \\
(\mathrm{n} / \mathrm{n})\end{array}$ & $\begin{array}{c}\text { Specificity, } \% \\
(\mathrm{n} / \mathrm{n})\end{array}$ & $\begin{array}{c}\text { Accuracy, } \% \\
(\mathrm{n} / \mathrm{n})\end{array}$ & $\begin{array}{c}\text { Negative predictive } \\
\text { value, } \%(\mathrm{n} / \mathrm{n})\end{array}$ & $\begin{array}{c}\text { Positive predictive } \\
\text { value, } \%(\mathrm{n} / \mathrm{n})\end{array}$ \\
\hline $\begin{array}{l}\text { TSH+FT4+ } \\
\text { gestational age }\end{array}$ & $11.66(80 / 686)$ & $92.86(65 / 70)$ & $97.56(601 / 616)$ & $97.08(666 / 686)$ & $99.17(601 / 606)$ & $81.25(65 / 80)$ \\
TSH+FT4 & $13.70(94 / 686)$ & $74.29(52 / 70)$ & $93.18(574 / 616)$ & $91.25(626 / 686)$ & $96.96(574 / 592)$ & $55.32(52 / 94)$ \\
$\chi^{2}$ & 1.290 & 8.792 & 20.293 & 21.238 & 7.806 & 13.193 \\
P-value & 0.256 & 0.003 & $<0.001$ & $<0.001$ & 0.005 & $<0.001$ \\
\hline
\end{tabular}

TSH, thyroid-stimulating hormone; FT4, free thyroxine. The numerator of negative predictive value is the numbers of true negative patients, and the denominator is the numbers of negative patients of TSH+FT4+ gestational age and TSH+FT4. The numerator of positive predictive value is the numbers of true positive patients, and the denominator is the numbers of positive patients of TSH+FT4+ gestational age and TSH+FT4. Therefore, the total numbers of the two groups are different.

Table III. Univariate analysis of the related risk factors of neonatal hypothyroidism.

\begin{tabular}{|c|c|c|c|c|c|}
\hline Risk factors & $\begin{array}{l}\text { Total number } \\
\text { of neonates, } n\end{array}$ & $\begin{array}{l}\text { Patient group, } \\
(\mathrm{n}=70) \mathrm{n}(\%)\end{array}$ & $\begin{array}{l}\text { Normal group, } \\
(\mathrm{n}=70) \mathrm{n}(\%)\end{array}$ & $\chi^{2}$ & P-value \\
\hline \multicolumn{6}{|l|}{ Sex } \\
\hline Male & 69 & $30(42.86)$ & $37(52.86)$ & \multirow[t]{2}{*}{1.403} & \multirow[t]{2}{*}{0.236} \\
\hline Female & 71 & $40(57.14)$ & $33(47.14)$ & & \\
\hline \multicolumn{6}{|c|}{ Gestational age, weeks } \\
\hline$<37$ & 31 & $26(34.1)$ & $5(7.14)$ & \multirow[t]{3}{*}{27.650} & \multirow[t]{3}{*}{$<0.001$} \\
\hline $37-42$ & 95 & $33(47.14)$ & $62(88.57)$ & & \\
\hline$>42$ & 14 & $11(15.71)$ & $3(4.29)$ & & \\
\hline \multicolumn{6}{|c|}{ Maternal thyroid function } \\
\hline Normal & 85 & $17(24.29)$ & $68(97.14)$ & \multirow[t]{2}{*}{77.891} & \multirow[t]{2}{*}{$<0.001$} \\
\hline Abnormal & 55 & $53(75.71)$ & $2(2.86)$ & & \\
\hline \multicolumn{6}{|c|}{ Fetal macrosomia $(>4,000 \mathrm{~g})$} \\
\hline Yes & 11 & $10(14.29)$ & $3(4.29)$ & \multirow[t]{2}{*}{4.155} & \multirow[t]{2}{*}{0.042} \\
\hline No & 129 & $60(85.71)$ & $67(95.71)$ & & \\
\hline \multicolumn{6}{|c|}{ Low birth weight $(<2,500 \mathrm{~g})$} \\
\hline Yes & 18 & $16(22.86)$ & $2(2.86)$ & \multirow[t]{2}{*}{12.495} & \multirow[t]{2}{*}{$<0.001$} \\
\hline No & 122 & $54(77.14)$ & $68(97.14)$ & & \\
\hline
\end{tabular}

According to the American College of Obstetricians and Gynecologists (AGOS) (31) in 2017, a newborn with a birth weight of $>4,500 \mathrm{~g}$ is considered as fetal macrosomia, and that with a birth weight of $<2,500 \mathrm{~g}$ is defined as low birth weight.

$>42$ weeks according to gestational age, and TSH and FT4 levels of neonates at different gestational ages were compared. TSH and FT4 levels of newborns with different gestational age were compared. The results showed that in the patient group, the gestational age at birth of 15 neonates was $<37$ weeks, 47 neonates was 37-42 weeks and 8 neonates was $>42$ weeks. The TSH levels of neonates $>42$ weeks of gestational age were significantly lower compared with neonates that were $37-42$ weeks and $<37$ weeks of gestational age $\left(\mathrm{F}_{\mathrm{TSH}}=10.772\right.$, $\mathrm{P}_{\mathrm{TSH}}<0.001$; Fig. 2A). The FT4 levels of neonates with $>42$ weeks of gestational age were significantly higher compared with neonates that were 37-42 weeks and $<37$ weeks of gestational age $\left(\mathrm{F}_{\mathrm{FT} 4}=15.468\right.$ and $\mathrm{P}_{\mathrm{FT} 4}<0.001$; Fig. 2B).
The TSH levels of neonates with 37-42 weeks of gestational age were significantly lower compared with neonates with $<37$ weeks of gestational age, while the FT4 levels of neonates with 37-42 weeks of gestational age were significantly higher compared with neonates $<37$ weeks of gestational age (Fig. 2). The aforementioned results suggest that the younger the gestational age at birth of neonates with hypothyroidism was, the more obvious the degree of hypothyroidism was.

Combined use of TSH plus FT4 levels and gestational age at birth had a higher predictive value compared with TSH plus FT4 levels in screening for neonatal hypothyroidism. Using a TSH level of $>9 \mathrm{mIU} / 1$, FT4 level of $<8.3 \mathrm{pmol} / \mathrm{l}$ and gestational 
Table IV. Logistic regression analyses of the risk factors for neonatal hypothyroidism.

$95 \% \mathrm{CI}$

\begin{tabular}{lcccccc}
\cline { 5 - 7 } Variables & $\beta$-value & Wald value & OR & P-value & Upper limit & Lower limit \\
\hline Gestational age & 2.157 & 8.338 & 0.811 & $<0.001$ & 1.180 & 1.272 \\
Low birth weight & 1.912 & 5.963 & 1.647 & $<0.001$ & 1.275 & 2.774 \\
Maternal thyroid dysfunction & 1.267 & 4.892 & 1.618 & 0.002 & 1.074 & 3.121
\end{tabular}

OR, odds ratio; $\mathrm{CI}$, confidence interval.

age at birth of $<37$ weeks as predictive values, the combined use of TSH+FT4 levels and gestational age at birth to predict neonatal hypothyroidism demonstrated a significantly higher positive predictive value, sensitivity and accuracy compared with the use of only TSH+FT4 levels $(\mathrm{P}<0.001$ vs. $\mathrm{P}=0.003$ vs. $\mathrm{P}<0.001$; Table II).

Univariate analyses of risk factors for neonatal hypothyroidism. Univariate analyses was performed and revealed that low gestational age at birth, maternal thyroid dysfunction, fetal macrosomia and low birth weight were the underlying risk factors for neonatal hypothyroidism $(\mathrm{P}<0.05$; Table III).

Low gestational age at birth, maternal thyroid dysfunction and low birth weight are risk factors for neonatal hypothyroidism. In logistics regression analysis, low gestational age at birth, maternal thyroid dysfunction, fetal macrosomia and low birth weight were included as dependent variables and neonatal hypothyroidism as the independent variable. The analysis revealed that low gestational age at birth, maternal thyroid dysfunction and low birth weight significantly increased the risk of neonatal hypothyroidism $\left(\mathrm{R}^{2}=0.375\right.$, $\mathrm{P}<0.001$; Table IV).

\section{Discussion}

The present study demonstrated that the patient group had a significantly lower gestational age at birth, lower birth weight, slower heart rate, lower FT4 level and higher TSH level compared with the control group. The patient group was supplemented with thyroxine for 8 weeks, following which TSH and FT4 levels significantly decreased and increased, respectively to normal levels. A previous study reported similar findings wherein the TSH and FT4 levels of neonates with thyroid dysfunction normalized following 4 weeks of thyroxine therapy and reached levels that were not significantly different compared with those of healthy controls (17). This indicated that treatment administered immediately following the diagnosis of neonatal hypothyroidism has the ability to normalize TSH and FT4 levels within a short period of time and effectively prevent clinical symptoms. The present study also demonstrated that gestational age at birth had a significant inverse association with TSH level, but had a direct association with levels of FT4. The reason for this may be because the thyroid gland developed further with increased gestational age at birth and reached the normal level. Neonates with at gesta- tional age of $<37$ weeks were born prematurely and their thyroid glands may have failed to develop normally compared with neonates of a gestational age $\geq 37$ weeks. Premature neonates were more likely to develop hypothyroidism with higher TSH and lower FT4 levels $(18,19)$. A relevant study indicated that the prevalence and positive screening rate of hypothyroidism are higher in premature neonates, which suggests that gestational age is closely related to hypothyroidism (20). In the present study, neonatal hypothyroidism was screened on the basis of TSH+FT4 levels in neonates with a gestational age at birth of $<37$ weeks and the results were compared with the screening results obtained using only TSH+FT4 levels. This comparison revealed that the combined use of TSH+FT4 levels and gestational age at birth was more effective in predicting neonatal hypothyroidism with significantly improved positive predictive value, sensitivity and accuracy. In the present study, the additional use of fetal gestational age increased the efficiency of early screening for thyroid function in neonates.

Analysis of the related risk factors for neonatal hypothyroidism revealed low gestational age at birth, maternal thyroid dysfunction and low birth weight to be risk factors. A previous study looking at the effects of gestational age at birth on neonatal hypothyroidism demonstrated that the fetal hypothalamus-pituitary-adrenal (HPA) axis was located at the center of the mechanisms controlling fetal growth and development by stimulating the production of a large amount of thyroid hormones in addition to performing the function of stabilizing their levels (21). When FT4 levels increased, the HPA axis performed negative feedback regulation to reduce TSH secretion (22). However, when FT4 levels decreased, the HPA axis increased TSH secretion (23). In addition, gestational age at birth was positively associated with HPA development and functions (24). The gestational age at birth was also demonstrated to be negatively associated with the detection rate of hypothyroidism (25). A study investigating the effects of maternal thyroid function on neonatal hypothyroidism demonstrated that in pregnant women, TSH failed to pass through the placental barrier while small amounts of TH3 and thyroxine successfully passed to the fetus. In addition, thyrotropin receptor antibodies passed through the placental barrier into the fetus, affecting the levels of neonatal thyroid hormones thereby causing neonatal thyroid dysfunction (26). Previous findings have revealed that thyroid dysfunction in pregnant women increases the rate of neonatal thyroid dysfunction (27). Maternal thyroid dysfunction may have been responsible for neonatal hypothyroidism in the present study. 
A study investigating the effects of birth weight on neonatal hypothyroidism demonstrated that low-birth-weight neonates were relatively underdeveloped; in addition, their HPA axis and thyroid glands were not well developed leading to hypothyroidism. These neonates were prone to non-thyroidal diseases and their thyroid function was affected due to a variable degree of growth retardation. In addition, they responded slowly to TSH changes and thyroid gland reactions and had poor thyroid hormone regulation (28). Due to limited iodine reserves and thyroglobulin content, low-birth-weight neonates had a negative iodine balance at early stages after birth and their iodine intake capacity was extremely low (29). This failed to effectively support the needs of neonatal growth and development increasing their risk of hypothyroidism (29). The weight of low-birth-weight neonates has also been reported to have more propensity for neonatal thyroid dysfunction (30), which is consistent with the results of the present study.

The present study had several limitations. Firstly, there were 2 and 3 premature neonates with gestational age at birth of $<37$ weeks in the control and patient groups, respectively. Even though other congenital diseases were excluded premature neonates have various problems that may interfere with their thyroid function, therefore the study results may be biased to a certain extent. As the present study was a preliminary study, future studies with larger cohorts excluding premature neonates are required to verify the findings of the present study. Secondly, when calculating the predictive value and identifying risk factors, the denominator was different. This may have been due to non-matched control recruitment with a small sample size due to inadequate birth weight and gestational age at birth. Future studies with a higher number of cases are required to eliminate the interference of the results of the aforementioned factors. Thirdly, in the present study the overall heart rate was relatively low and the average heart rate of the neonates was 97. Despite various possible neonatal conditions, such as bradycardia being ruled out in the present study severe hypothyroidism may be the cause of the low heart rate detected in the present study. Future studies with larger sample sizes are needed for the change in heart rate to be further assessed. Future large scale studies are required to analyze the predictive value of these indicators for neonates with different degrees of hypothyroidism and premature neonates with hypothyroidism. Furthermore, the study duration was short (8 weeks). Neonates with hypothyroidism were not followed up for long-term thyroid function. Although neonates with hypothyroidism were actively treated and thyroid function gradually returned to normal, the long-term effects on children have not been analyzed. This could be the focus of a future study. In the present study, maternal thyroid dysfunction was not fully described i.e. whether it was subclinical hypothyroidism, Hashimoto's thyroiditis, hyperthyroidism or otherwise was not elaborated. Future studies could investigate the effects of maternal thyroid dysfunction on neonates.

In conclusion, in the present study TSH and FT4 levels increased and decreased, respectively in neonates with hypothyroidism aged $<3$ days; these values normalized following treatment with thyroxine. Gestational age at birth was negatively associated with the TSH level, but was positively associated with the FT4 level. The combined use of TSH+FT4 levels and gestational age at birth is an improved predictor of neonatal hypothyroidism and helps in early treatment and intervention. Low gestational age at birth, maternal thyroid dysfunction and low birth weight increased the risk of neonatal hypothyroidism; therefore, special intervention should be administered to pregnant women with thyroid dysfunction to reduce the incidence of neonatal hypothyroidism. Based on the findings of the present study, pre-pregnancy screening is strongly advocated especially for women planning for pregnancy who have had thyroid dysfunction or have a family history of thyroid disease.

\section{Acknowledgements}

Not applicable.

\section{Funding}

No funding was received.

\section{Availability of data and materials}

The datasets used and/or analyzed during the current study are available from the corresponding author on reasonable request.

\section{Authors' contributions}

JL wrote the manuscript. JL, JC and QL designed the study, collected the data and performed the statistical analyses. All authors have read and approved the manuscript.

\section{Ethics approval and consent to participate}

The present study was approved by the Ethics Committee of The First Affiliated Hospital of Chongqing Medical University (Chonqing, China). The parents of the neonates provided written consent for study participation.

\section{Patient consent for publication}

Not applicable.

\section{Competing interests}

The authors declare that they have no competing interests.

\section{References}

1. Sarkar A, Sar P and Islam E: Hexavalent chromium reduction by microbacterium oleivorans A1: A possible mechanism of chromate-detoxification and -bioremediation. Recent Pat Biotechnol 9: 116-129, 2016.

2. Khordad E, Alipour F, Beheshti F, Hosseini M, Rajabzadeh AA, Asiaei $\mathrm{F}$ and Seghatoleslam M: Vitamin C prevents hypothyroidism associated neuronal damage in the hippocampus of neonatal and juvenile rats: A stereological study. J Chem Neuroanat 93: 48-56, 2018.

3. Zhu WB, Chen HQ, Wang J, Lin F, Zhao H and Zhang HH: Study on the cut-off value of TSH screening for neonatal congenital hypothyroidism in Fujian Province. Chin J Child Health Care 11: $13,2003$.

4. Tian JR: Study on the cut-off value of TSH screening for congenital hypothyroidism in neonates. China Health Care Nutr 29: 124, 2019. 
5. Rahmani K, Yarahmadi S, Etemad K, Mehrabi Y, Aghang N, Koosha A and Soori H: Intelligence quotient at the age of six years of Iranian children with congenital hypothyroidism. Indian Pediatrics 55: 121-124, 2018

6. Xu YH, Qin YF and Zhao ZY: Retrospective study on neonatal screening for congenital hypothyroidism and phenylketonuria in China in the past 22 years. Zhonghua Er Ke Za Zhi 47: 18-22, 2009 (In Chinese)

7. García M, Barrio R, García-Lavandeira M, Garcia-Rendueles AR, Escudero A, Díaz-Rodríguez E, Del Blanco DG, Fernández A, De Rijke YB, Vallespín E, et al: The syndrome of central hypothyroidism and macroorchidism: IGSF1 controls TRHR and FSHB expression by differential modulation of pituitary TGF $\beta$ and Activin pathways. Sci Rep 7: 42937, 2017.

8. Dayal D, Giri D and Senniappan S: A rare association of central hypothyroidism and adrenal insufficiency in a boy with Williams-Beuren syndrome. Ann Pediatr Endocrinol Metab 22: 65-67, 2017.

9. Zhou W and Xi X: Screening of congenital hypothyroidism and early intervention effect of levothyroxine. China Pharm 21 81-82, 2012

10. Aubry G, Pontvianne M, Chesnais M, Weingertner AS, Guerra F and Favre R: Prenatal diagnosis of fetal Goitrous hypothyroidism in a Euthyroid mother: A management challenge. J Ultrasound Med 36: 2387-2392, 2017.

11. Oguma M, Kobayashi M, Yamazaki M, Yokoyama K, Morikawa S, Yamaguchi T, Yamagata T and Tajima T: Two siblings with congenital central hypothyroidism caused by a novel mutation in the IGSF1 gene. Clin Pediatr Endocrinol 27: 95-100, 2018.

12. Siffo S, Adrover E, Citterio CE, Miras MB, Balbi VA, Chiesa A, Weill J, Sobrero G, González VG, Papendieck P, et al: Molecular analysis of thyroglobulin mutations found in patients with goiter and hypothyroidism. Mol Cell Endocrinol 473: 1-16, 2018.

13. Thaker VV, Galler MF, Marshall AC, Almodovar MC, Hsu HW, Addis CJ, Feldman HA, Brown RS and Levine BS: Hypothyroidism in infants with congenital heart disease exposed to excess iodine. J Endocr Soc 1: 1067-1078, 2017.

14. Zhang CP: Correlation analysis of high thyroid stimulating hormone outcome in newborns and congenital hypothyroidism. Guangzhou Med J 2: 88-90, 2019.

15. Tsagarakis S, Tzanela M and Dimopoulou I: Diabetes insipidus, secondary hypoadrenalism and hypothyroidism after traumatic brain injury: Clinical Implications. Pituitary 8: 251-254, 2005

16. Stagnaro-Green A, Abalovich M, Alexander E, Azizi F, Mestman J, Negro R, Nixon A, Pearce EN, Soldin OP, Sullivan S, et al: Guidelines of the American Thyroid Association for the diagnosis and management of thyroid disease during pregnancy and postpartum. Thyroid 21: 1081-1125, 2011.

17. Fernandez Rodriguez B and Perez Diaz AJ: Evaluation of a follow up protocol of infants born to mothers with antithyroid antibodies during pregnancy. J Matern Fetal Neonatal Med 31: 312-319, 2018.

18. Asiaei F, Fazel A, Rajabzadeh AA, Hosseini M, Beheshti F and Seghatoleslam M: Neuroprotective effects of Nigella sativa extract upon the hippocampus in PTU-induced hypothyroidism juvenile rats: A stereological study. Metab Brain Dis 32: $1755-1765,2017$
19. Kobayashi M, Yagasaki H, Saito T, Nemoto A, Naito A and Sugita K: Fetal goitrous hypothyroidism treated by intra-amniotic levothyroxine administration: Case report and review of the literature. J Pediatr Endocrinol Metab 30: 1001-1005, 2017.

20. McGrath N, Hawkes CP, McDonnell CM, Cody D, O'Connell S, Mayne PD and Murphy NP: Incidence of congenital hypothyroidism over 37 years in Ireland. Pediatrics 142: e20181199, 2018.

21. Onal Z, Balkaya S, Ersen A, Mutlu N, Onal H and Adal E: Possible effects of neonatal vitamin B12 status on TSH-screening program: A cross-sectional study from Turkey. J Pediatr Endocrinol Metab 30: 551-555, 2017.

22. Camargo Neto E, Schulte J, Pereira J, Bravo H, Sampaio-Filho C and Giugliani R: Neonatal screening for four lysosomal storage diseases with a digital microfluidics platform: Initial results in Brazil. Genet Mol Biol 41: 414-416, 2018.

23. Valizadeh M, Nazeri P, Fazli F, Mohammadian F, Kalantari S, Kamali $\mathrm{K}$ and Osali $\mathrm{H}$ : Application of povidone-iodine at delivery significantly increases maternal urinary iodine but not neonatal thyrotropin in an area with iodine sufficiency. J Pediatr Endocrinol Metab 30: 967-972, 2017.

24. Braslavsky D, Mendez MV, Prieto L, Keselman A, Enacan R, Gruneiro-Papendieck L, Jullien N, Savenau A, Reynaud R, Brue $\mathrm{T}$, et al: Pilot neonatal screening program for central congenital hypothyroidism: Evidence of significant detection. Horm Res Paediatr 88: 274-280, 2017.

25. Sarkar D and Singh SK: Neonatal hypothyroidism affects testicular glucose homeostasis through increased oxidative stress in prepubertal mice: Effects on GLUT3, GLUT8 and Cx43. Andrology 5: 749-762, 2017.

26. Lara NLM and Franca LR: Neonatal hypothyroidism does not increase Sertoli cell proliferation in iNOS(-/-) mice. Reproduction 154: 13-22, 2017.

27. Akangire G, Cuna A, Lachica C, Fischer R, Raman S and Sampath V: Neonatal Graves' disease with maternal hypothyroidism. AJP Rep 7: e181-e184, 2017.

28. Chen JG, Zhong YH, Cai XJ, Ye LX and Xie CL: Effects of birth weight on thyroid stimulating hormone levels and incidence of congenital hypothyroidism in neonates. Medical Information 30: 52-53, 2017.

29. Leeuwen L, van Heijst AF, Vijfhuize S, Beurskens LW, Weijman G, Tibboel D, van den Akker EL and Ijsselstijn H: Nationwide evaluation of congenital hypothyroidism screening during neonatal extracorporeal membrane oxygenation. Neonatology 111: 93-99, 2017.

30. Novakovic TR, Dolicanin ZC and Djordjevic NZ: Oxidative stress biomarkers in amniotic fluid of pregnant women with hypothyroidism. J Matern Fetal Neonatal Med 32: 1105-1110, 2019.

31. Qi HB and Zheng J: ACOG Guidelines for management of fetal macrosomia: Essential introduction. J Chongqing Med Univ 42: 925-928, 2017 (In Chinese). 